\title{
Exploring the mechanism of fracture for entangled polymer liquids in extensional flow
}

\author{
Huang, Qian
}

Published in:

Physics of Fluids

Link to article, DOI:

$10.1063 / 1.5108510$

Publication date:

2019

Document Version

Publisher's PDF, also known as Version of record

Link back to DTU Orbit

Citation (APA):

Huang, Q. (2019). Exploring the mechanism of fracture for entangled polymer liquids in extensional flow. Physics of Fluids, 31(8), [083105]. https://doi.org/10.1063/1.5108510

\section{General rights}

Copyright and moral rights for the publications made accessible in the public portal are retained by the authors and/or other copyright owners and it is a condition of accessing publications that users recognise and abide by the legal requirements associated with these rights.

- Users may download and print one copy of any publication from the public portal for the purpose of private study or research.

- You may not further distribute the material or use it for any profit-making activity or commercial gain

- You may freely distribute the URL identifying the publication in the public portal

If you believe that this document breaches copyright please contact us providing details, and we will remove access to the work immediately and investigate your claim. 


\section{Exploring the mechanism of fracture for entangled polymer liquids in extensional flow $\oplus^{\circ}$}

Cite as: Phys. Fluids 31, 083105 (2019); https://doi.org/10.1063/1.5108510

Submitted: 29 April 2019 . Accepted: 01 August 2019 . Published Online: 20 August 2019

Qian Huang (D)

COLLECTIONS

Note: This paper is part of the special topic on Invited Contributions from Outstanding Early Career Researchers.

EP This paper was selected as an Editor's Pick
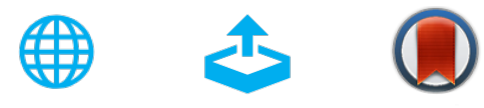

\section{ARTICLES YOU MAY BE INTERESTED IN}

Effects of bulk viscosity on compressible homogeneous turbulence

Physics of Fluids 31, 085115 (2019); https://doi.org/10.1063/1.5111062

The rising velocity of a slowly pulsating bubble in a shear-thinning fluid

Physics of Fluids 31, 083103 (2019); https://doi.org/10.1063/1.5108812

Time-dependent study of anisotropy in Rayleigh-Taylor instability induced turbulent flows with a variety of density ratios

Physics of Fluids 31, 084106 (2019); https://doi.org/10.1063/1.5110914

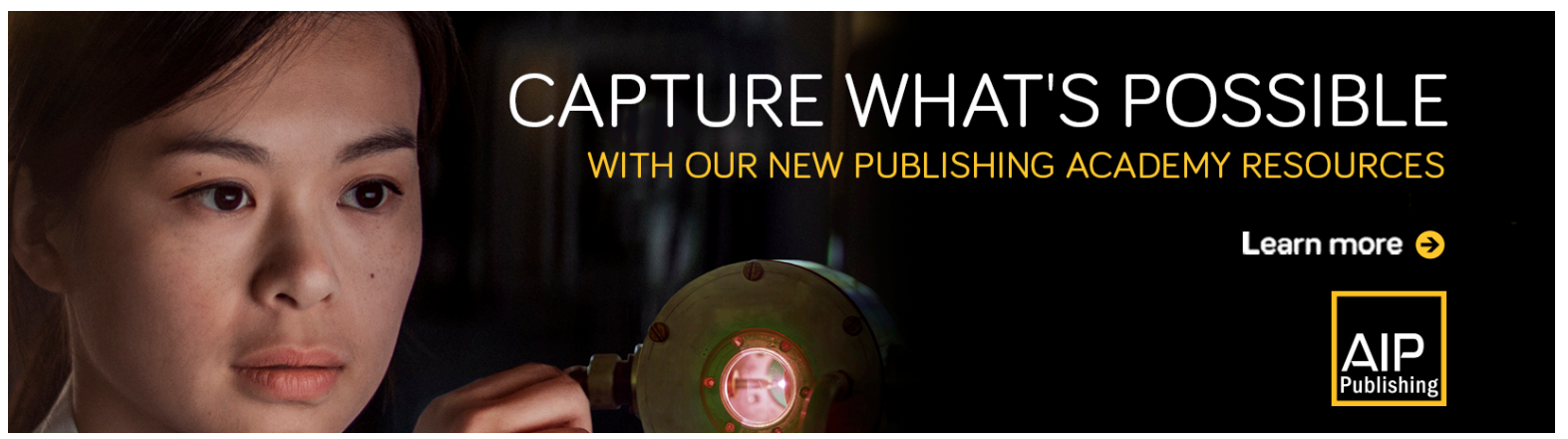




\title{
Exploring the mechanism of fracture for entangled polymer liquids in extensional flow
}

\author{
Cite as: Phys. Fluids 31, 083105 (2019); doi: 10.1063/1.5108510 \\ Submitted: 29 April 2019 • Accepted: 1 August 2019 • \\ Published Online: 20 August 2019
}

Qian Huang

AFFILIATIONS

Department of Chemical and Biochemical Engineering, Technical University of Denmark, DK-2800 Kgs. Lyngby, Denmark

Note: This paper is part of the special topic on Invited Contributions from Outstanding Early Career Researchers.

a)Email: qh@kt.dtu.dk

\begin{abstract}
The critical strain and stress at fracture are systematically investigated for two groups of nearly monodisperse linear polystyrene liquids in an extensional flow. The samples in group I have similar number of Kuhn segments per entangled strand $\left(N_{e}\right)$ but different number of entanglements per chain $(Z)$, while the samples in group II have similar $Z$ but different $N_{e}$. We found that the critical conditions, especially the critical stress, are independent of $Z$ but influenced by $N_{e}$. The observations indicate that the fracture in entangled polystyrene liquids occurs at a length scale smaller than an entangled strand. Therefore, the fracture originates more likely from scission of primary bonds in polymer chains, rather than rapid entanglement slipping. The level of the critical stress also suggests that at fracture, the polymer chains approach their theoretical maximum stretch ratio, which is equal to $\sqrt{N_{e}}$.
\end{abstract}

Published under license by AIP Publishing. https://doi.org/10.1063/1.5108510

\section{INTRODUCTION}

Fracture is a common phenomenon in solid materials. It limits the strength of the material. In general, the initiation of fracture is related to the effect of local instability. In many solid materials, this local instability is due to defects in terms of microcracks, and the biggest pre-existing microcrack determines the fracture stress. Since the size of the biggest crack varies, the fracture stress is seldom reproducible. Therefore, fracture stress is not a material property for solids. In respect of glassy polymers, it has been found that the tensile strength is also influenced by the alignment of the polymer chains during processing. ${ }^{2,3}$ In a recent paper by Huang et al., ${ }^{3}$ it has been observed that for polystyrene, the larger stress in the melt stretching gives the larger tensile strength in the solid state, while the largest stress in the melt stretching is limited by fracture of the polymer liquid during stretching. This observation indicates that there is a connection between liquid fracture and solid fracture for polymer materials. While the explanation based on pre-existing cracks works for solid fracture, it does not seem compatible with liquids where such microcracks would close up by molecular motion. ${ }^{4-6}$ Thus, understanding why polymers fracture in the liquid state promises to hold the key to understand how the inherent strength of defect-free materials depends on molecular parameters.

Entangled polymer liquids such as polymer melts and solutions are known to be viscoelastic materials, meaning that they react liquidlike in slow deformations and solidlike in fast deformations. They are able to fracture in a manner similar to solids at sufficiently high deformation rates in an extensional flow. Fracture in polymer liquids has been observed in extrusion processes ${ }^{7,8}$ and in the breakage of filaments. ${ }^{9,10}$ It should be noted that the fracture mentioned here is fundamentally different from the phenomena of flow instabilities such as necking and surface tension-driven breakup (e.g., beads-on-a-string ${ }^{11}$ that may be described using the basic equations of fluid mechanics and macroscopic constitutive equations. ${ }^{12}$ "Edge fracture" in shear deformations is also a phenomenon of flow instability. ${ }^{13}$ By contrast, the phenomenon of fracture should rely on some molecular (i.e., noncontinuum) process for its initiation and propagation. This may happen in a fast flow with a sudden brittlelike fracture, which breaks the sample into two pieces with new smooth surfaces. ${ }^{4-6}$ Malkin and co-workers ${ }^{10,14,15}$ proposed a master curve dividing the behavior of polymer liquids into four zones by plotting the Hencky strain at the rupture as a function of stretch rate. The master curve shows that a steady state flow can be observed at very 
slow rates in Zone I, while necking instabilities take place at faster rates in Zones II and III. At very high rates in Zone IV, the materials fracture in a solidlike fashion. The fracture discussed in this paper corresponds to the behavior in Zone IV of the Malkin master curve.

Current theoretical and experimental information on the mechanism of fracture in polymer liquids is very limited. Pomeau introduced the idea that thermal fluctuations may play a role in fracture, ${ }^{16}$ and this idea was first used for viscoelastic materials by Bonn et al. ${ }^{17}$ de Gennes suggested a simple viscoelastic trumpet model to describe crack propagation in viscoelastic fluids. ${ }^{18}$ However, these theories have largely remained without experimental verification. In 2009, Tabuteau and co-workers provided the first pictures of a crack propagating in the trailing liquid filament behind a falling drop, using high-speed imaging. ${ }^{4}$ The images demonstrate that the fracture in polymer liquids involves crack initiation from the sample surface and crack propagation, in contrast to the axisymmetric flow instabilities such as necking and beads-on-a-string. Unfortunately, Tabuteau et al. were unable to control the rate of stretching of the liquid filament. Therefore, they were unable to investigate how the fracture process depends on the stretch rate. Recent developments in extensional rheometers make it possible to stretch liquid filaments at controlled stretch rates. ${ }^{19}$ Carefully controlled experiments have shown that the critical stress and strain at the fracture for polymer liquids are highly reproducible. ${ }^{6}$ This observation is nearly impossible in the solid fracture, suggesting that the liquid fracture must be understood from a fundamentally different framework than classical solid fracture mechanics. This formulates the following key question: what is the underlying molecular mechanism for liquid fracture? Is it scission of primary bonds or is it just a very rapid disentanglement process? While there is no clear answer at this stage, it has been suggested by some research groups that scission of primary bonds plays the role. ${ }^{20,21}$ Very recently, Wagner et al. proposed that liquid fracture should be understood in terms of entropy. ${ }^{21}$ However, this idea has been questioned by Wang. ${ }^{22}$

Although fracture stops the flow of polymer liquids and should be understood as a noncontinuum process, the conditions that lead to fracture, e.g., stretch rate, critical strain, and critical stress, are still connected to polymer rheology and dynamics. Commonly known as the tube theory, the seminal developments by de Gennes ${ }^{23}$ as well as by Doi and Edwards ${ }^{24}$ provide the basis for the molecular picture of the dynamics of entangled polymer liquids. There are two important molecular parameters in the tube model: the number of entanglements per chain $(Z)$ and the number of Kuhn segments per entangled strand $\left(N_{e}\right)$. The significance of $Z$ is that the total frictional resistance to rapid entanglement sliding must presumably depend on $Z$. The significance of $N_{e}$ is that it controls the maximum stretch ratio for one entanglement strand.

In this work, we systematically investigate the critical strain and critical stress for a series of nearly monodisperse entangled polymer liquids with different $Z$ and $N_{e}$. The purpose is to provide clearer indications for the key questions mentioned above. If the fracture originates from rapid entanglement slipping, the critical conditions at the fracture should be dependent on $Z$. If the polymer chains are fully stretched at the fracture, the critical conditions should scale with $N_{e}$.

\section{MATERIALS AND METHODS}

\section{A. Materials}

Two groups of polystyrene (PS) samples have been prepared for this study. The samples are named in the form of PS-X/4k-Y, where $\mathrm{X}$ is the weight average molecular weight of the PS, $4 \mathrm{k}$ is the molecular weight of the oligomeric styrene solvent, and Y\% is the volume fraction of the PS in the solution. Group I contains three PS solutions with similar volume fractions $(17 \%-18 \%)$ of the polymer, namely, PS-1760k/4k-18, PS-864k/4k-17, and PS-576k/4k-17. The samples have similar values of $N_{e}$ but different $Z$. Group II contains three PS samples with different volume fractions (18\%, 33\%, and 100\%), namely, PS-1760k/4k-18, PS-864k/4k-33, and PS-285k. The samples have similar values of $Z$ but different $N_{e}$. All the polystyrenes for making the solutions were purchased from Polymer Standards Service (PSS). They were separately diluted in a styrene oligomer, OS-4k. The OS-4k has a short chain far below the entanglement molecular weight, and is therefore equivalent to a solvent. The purpose of selecting a styrene oligomer as the solvent is to avoid potential phase separation. The detailed procedure of making the solutions has been described in Ref. 25. The polystyrene melt, PS-285k, was synthesized using anionic polymerization. All the samples are nearly monodisperse, with dispersity below 1.15 . Some of the samples have been used in previous work and the corresponding references have been listed in Table I. The values of $Z$ and $N_{e}$, together with the other parameters such as the glass transition temperature $\left(T_{g}\right)$, the relaxation time of one entanglement strand $\left(\tau_{c}\right)$, the maximum relaxation time $\left(\tau_{m}\right)$, the plateau modulus $\left(G_{N}^{0}\right)$, and the maximum stretch ratio of the polymer chain $\left(\lambda_{\max }\right)$, are also listed in Table I. The values of $N_{e}$ are calculated from $N_{e}=N_{e, m} \phi^{-1}$, where $\phi$ is the volume fraction of the polymer and $N_{e, m}=21.8$ is the number of Kuhn segments

TABLE I. Material properties of the polystyrene samples.

\begin{tabular}{|c|c|c|c|c|c|c|c|c|c|}
\hline & Sample & $T_{g}\left({ }^{\circ} \mathrm{C}\right)$ & $N_{e}$ & $Z$ & $\tau_{c}(\mathrm{~s})\left(\right.$ at $\left.130^{\circ} \mathrm{C}\right)$ & $\tau_{m}(\mathrm{~s})\left(\right.$ at $\left.130^{\circ} \mathrm{C}\right)$ & $G_{N}^{0}(\mathrm{~Pa})\left(\right.$ at $\left.130{ }^{\circ} \mathrm{C}\right)$ & $\lambda_{\max }$ & References \\
\hline & PS-576k/4k-17 & 89.0 & 128.2 & 6.7 & 0.683 & 199.4 & 6890 & 11.3 & \\
\hline \multirow[t]{2}{*}{ I } & PS-864k/4k-17 & $89.0^{\mathrm{a}}$ & 128.2 & 10.2 & 0.757 & 926.2 & 6690 & 11.3 & 6 \\
\hline & PS-1760k/4k-18 & $89.0^{\mathrm{a}}$ & 121.1 & $22.8^{\mathrm{a}}$ & $0.661^{\mathrm{a}}$ & $12615^{\mathrm{a}}$ & $6850^{a}$ & 11.0 & 27 \\
\hline \multirow[t]{2}{*}{ II } & PS-864k/4k-33 & $92.0^{\mathrm{a}}$ & 66.1 & 21.0 & 0.391 & 5651 & 25942 & 8.1 & 6 \\
\hline & PS-285k & $107.5^{\mathrm{a}}$ & $21.8^{\mathrm{a}}$ & $21.4^{\mathrm{a}}$ & $0.444^{\mathrm{a}}$ & $6846^{\mathrm{a}}$ & $252040^{\mathrm{a}}$ & $4.7^{\mathrm{a}}$ & 25 \\
\hline
\end{tabular}

${ }^{\mathrm{a}}$ Values are directly taken from the corresponding references. 
per entangled strand for the PS melt. ${ }^{25}$ The values of $\lambda_{\max }$ are calculated as $\lambda_{\text {max }}=\sqrt{N_{e}}$. The values of $\tau_{c}, \tau_{m}$, and $G_{N}^{0}$ are obtained from fitting the Baumgaertel-Schausberger-Winter (BSW) model $^{26}$ to linear viscoelastic characterization data as described in Ref. 25.

\section{B. Linear viscoelastic characterization}

The linear viscoelastic properties of the PS samples were obtained from small amplitude oscillatory shear flow measurements using an ARES-G2 rheometer from TA Instruments. An $8 \mathrm{~mm}$ plate-plate geometry (with a sample thickness around $0.8 \mathrm{~mm}$ ) was used for lower frequencies and a $4 \mathrm{~mm}$ plate-plate geometry (with a sample thickness around $0.5 \mathrm{~mm}$ ) was used for higher frequencies in the transition to the glassy regime. The measurements were performed at $5{ }^{\circ} \mathrm{C}$ (for glassy regime), to $80^{\circ} \mathrm{C}$ higher than the corresponding glass transition temperatures of the samples. For each sample, the data were shifted to a single master curve at the reference temperature of $130^{\circ} \mathrm{C}$ using the time-temperature superposition procedure.

\section{Extensional stress-strain measurements}

The stress-strain measurements in a uniaxial extensional flow were performed using a homemade filament stretching rheometer (FSR). ${ }^{19}$ The initial shapes of the samples were cylindrical test specimens with a radius $R_{0}$ between $2.7 \mathrm{~mm}$ and $4.0 \mathrm{~mm}$ and a height $L_{0}$ between $1.5 \mathrm{~mm}$ and $2.0 \mathrm{~mm}$. To avoid sample slipping off the end plates during stretching, all the cylindrical samples were prestretched to a radius $R_{p}\left(<R_{0}\right)$ at the midfilament plane prior to the measurements. During the measurements, the force $F(t)$ was measured by using a load cell and the diameter $2 R(t)$ at the midfilament plane was measured by using a laser micrometer. The Hencky strain is defined as $\epsilon(t)=-2 \ln \left(R(t) / R_{p}\right)$, and the true stress is calculated from the measured force divided by the cross-sectional area of the midfilament plane. An online control scheme ${ }^{19}$ was employed in the FSR to control the midfilament diameter during stretching, so that the local strain rate, $\dot{\epsilon}=\mathrm{d} \epsilon / \mathrm{d} t$, was ensured to be constant. It should be noted that necking instabilities in Zones II and III in the Malkin master curve $e^{10,14,15}$ can be controlled by the online control scheme in the FSR. Therefore, the four zones in the Malkin master curve are reduced to just two states: steady flow and elastic fracture. ${ }^{28}$ All the measurements were performed at $120^{\circ} \mathrm{C}-130^{\circ} \mathrm{C}$, and shifted to the reference temperature of $130{ }^{\circ} \mathrm{C}$.

\section{RESULTS AND DISCUSSIONS}

\section{A. Linear viscoelasticity}

Figure 1(a) shows the storage modulus $G^{\prime}$ and loss modulus $\mathrm{G}^{\prime \prime}$ as a function of the angular frequency $\omega$ at the reference temperature $130{ }^{\circ} \mathrm{C}$ for the three samples in group I, while Fig. 1(b) plots the phase angle $\delta$ as a function of the complex modulus $\mathrm{G}^{*}$, which is known as the van Gurp-Palmen plot. ${ }^{29}$ The samples in group I are supposed to have similar values of $N_{e}$ but different values of $Z$. This can be checked in the figures. In Fig. 1(b), it is clear that the minimum of $\delta$ for the three samples appears at a similar value of $\mathrm{G}^{*}$, meaning that they have the similar plateau modulus $G_{N}^{0} \cdot{ }^{30}$ Since $G_{N}^{0} \propto M_{e}^{-1}$, where $M_{e}$ is the entanglement molecular weight, the similar $G_{N}^{0}$ also means that the samples have the similar $M_{e}$, hence
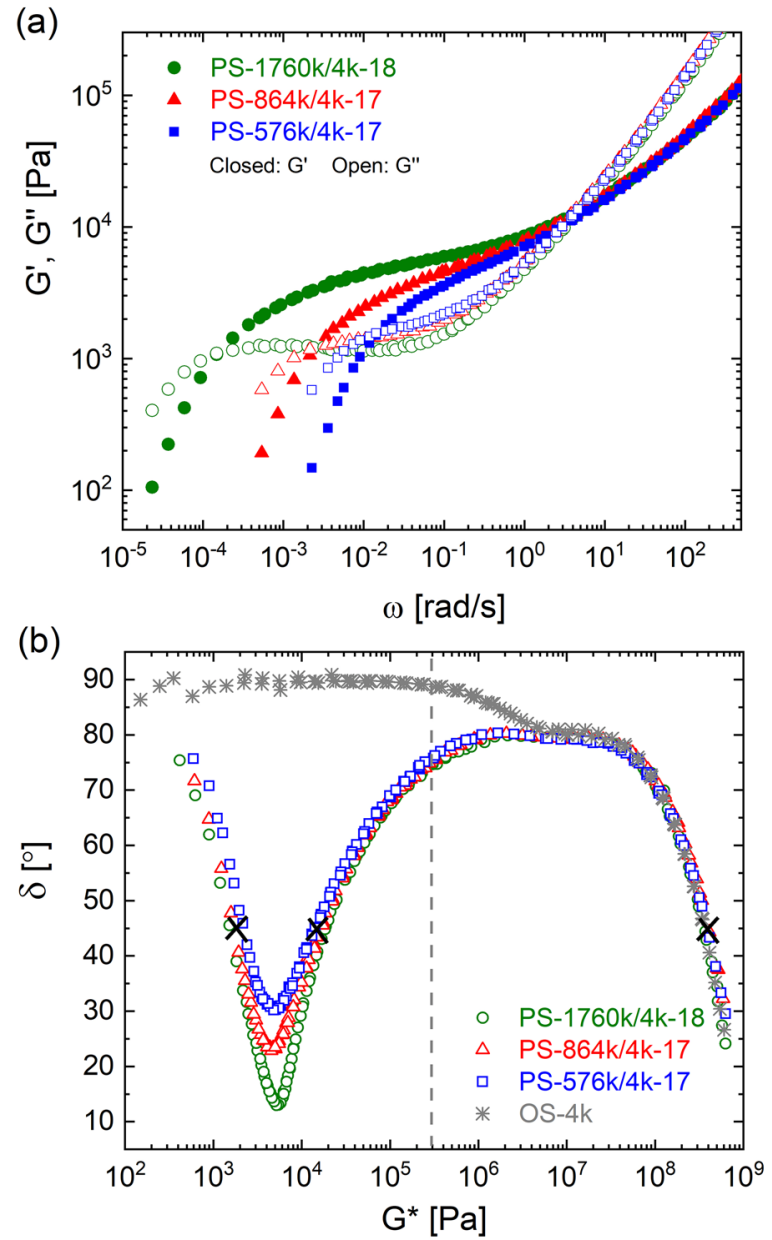

FIG. 1. (a) The storage modulus $G^{\prime}$ and loss modulus $G^{\prime \prime}$ as a function of the angular frequency $\omega$ at $130^{\circ} \mathrm{C}$ for group I. (b) The van Gurp-Palmen plots (phase angle vs complex modulus) for group I. The three black crosses $\left(\delta=45^{\circ}\right)$ correspond to the three crossover points $\left(G^{\prime}=G^{\prime \prime}\right)$ for each sample in Fig. 1 (a) [the part of $G^{*}>3 \times 10^{5} \mathrm{~Pa}$ in Fig. $1(\mathrm{~b})$ is not shown in Fig. 1(a)].

the similar $N_{e}$. The three black crosses (at $\delta=45^{\circ}$ ) in Fig. 1(b) correspond to the three crossover points $\left(G^{\prime}=G^{\prime \prime}\right)$ for each sample in Fig. 1(a) [the third crossover is not shown in Fig. 1(a)]. The part in between the second and third crosses in Fig. 1(b) for the three samples overlaps each other, also showing that the samples have the similar $N_{e}{ }^{31}$ The different values of the minimum $\delta$ in Fig. 1(b) show that the samples have different $Z{ }^{30}$ This is also confirmed in Fig. 1(a), where the horizontal distance between the first and second crossover points is different. ${ }^{25,31}$

Figure 2 shows the same plots as Fig. 1 but for the three samples in group II. These samples are supposed to have similar values of $Z$ but different values of $N_{e}$. In Fig. 2(b), the minimum of $\delta$ for the three samples appears at different $G^{*}$, meaning that they have different $G_{N}^{0}$, hence different $N_{e}$. This is also confirmed in Fig. 2(a), where the horizontal distance between the second and third crossover points is different for the three samples. The similar 

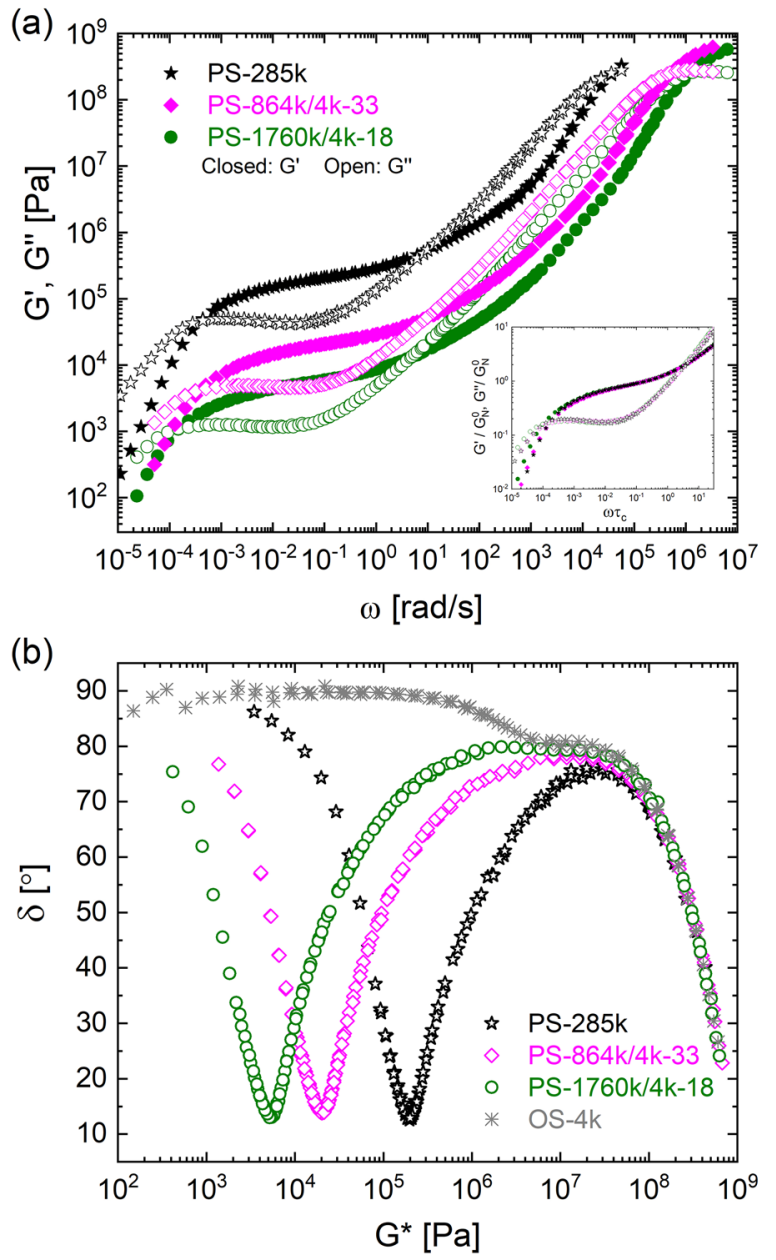

FIG. 2. (a) The storage modulus $G^{\prime}$ and loss modulus $G^{\prime \prime}$ as a function of the angular frequency $\omega$ at $130^{\circ} \mathrm{C}$ for group II. The inset shows the nondimensional form of the data, in which the same horizontal distance between the first and second crossover points is clearer. (b) The corresponding van Gurp-Palmen plots (phase angle vs complex modulus).

values of the minimum $\delta$ in Fig. 2(b) show that the samples have similar values of $Z$. This is also confirmed in Fig. 2(a), where the horizontal distance between the first and second crossover points is similar.

The plots of $\mathrm{G}^{\prime}$ and $\mathrm{G}^{\prime \prime}$ up to the frequency of the second crossover point have been previously published for PS-285k, PS-864k/4k-33, PS-1760k/4k-18, and PS-864k/4k-17 (see Table I for references). Here, these data have been remeasured and they agree with the published data. The $\mathrm{G}^{\prime}$ and $\mathrm{G}^{\prime \prime}$ data up to the second crossover point have been fitted with the BSW model ${ }^{26}$ for all the samples, following the same procedure as described in Refs. 25 and 27. The fitted values of $\tau_{c}, \tau_{m}$, and $G_{N}^{0}$ are listed in Table I. The details about the meaning of the BSW parameters and their relation to tube model parameters are explained in Ref. 25. The values of $Z$ in Table I are obtained from the relation $\tau_{m} / \tau_{c} \propto Z^{3.4} \cdot{ }^{25,27}$ The Rouse time for each sample is estimated as $\tau_{R}=Z^{2} \tau_{c}$. ${ }^{25}$ Figure 1 (b) also plots the contribution of the oligomer solvent OS-4k. It acts as a solvent up to $G^{*} \approx 3 \times 10^{5} \mathrm{~Pa}$.

\section{B. Critical strain and stress for group I (similar $N_{e}$ and different $Z$ )}

Figure 3 shows the transient true stress during stretching as a function of Hencky strain at different stretch rates at $130^{\circ} \mathrm{C}$ for one of the samples (PS-1760k/4k-18) as an example. For the rates faster than $1.0 \mathrm{~s}^{-1}$, the measurements were originally performed at $125^{\circ} \mathrm{C}$ or $120^{\circ} \mathrm{C}$, and shifted to $130^{\circ} \mathrm{C}$. It can be seen that for the measurements at low stretch rates, steady stress is achieved at high Hencky strain values, as shown by the plots with closed symbols in the figure. For these measurements, the samples did not fracture during stretching. The reason for terminating the measurements was that the diameter of the stretched filament was too thin to be measured accurately. By contrast, at high stretch rates, the samples fractured during stretching and thus terminated the measurements, as shown by the plots with open symbols in the figure.

Figure 4(a) plots the critical Hencky strain at the fracture as a function of stretch rates at $130^{\circ} \mathrm{C}$ for all the three samples in group I. At very low rates in which the samples did not fracture, the critical Hencky strain is infinite, and thus not plotted in the figure. It seems that when the stretch rate is high enough, the critical Hencky strain is the same for the three samples, meaning that it is independent of $Z$. At lower stretch rates, it seems that the critical Hencky strain is $Z$-dependent. If the fractures in this regime were due to rapid entanglement slipping, we would expect the sample with the lowest $Z$ (PS$576 / 4 \mathrm{k}-17$ ) to be the easiest to fracture. However, the figure shows the opposite case: PS-576/4k-17 requires a higher stretch rate to fracture. This is probably because the relaxation time for PS-576/4k-17 is smaller.

We therefore replot the critical Hencky strain as a function of the normalized stretch rates in Fig. 4(b). The normalized stretch rate is expressed in terms of the Weissenberg number, $W i_{R}=\dot{\epsilon} \tau_{R}$, where $\tau_{\mathrm{R}}$ is the Rouse time obtained from $\tau_{R}=Z^{2} \tau_{c}$. It can be seen now that for all the rates measured, the critical Hencky strain is almost

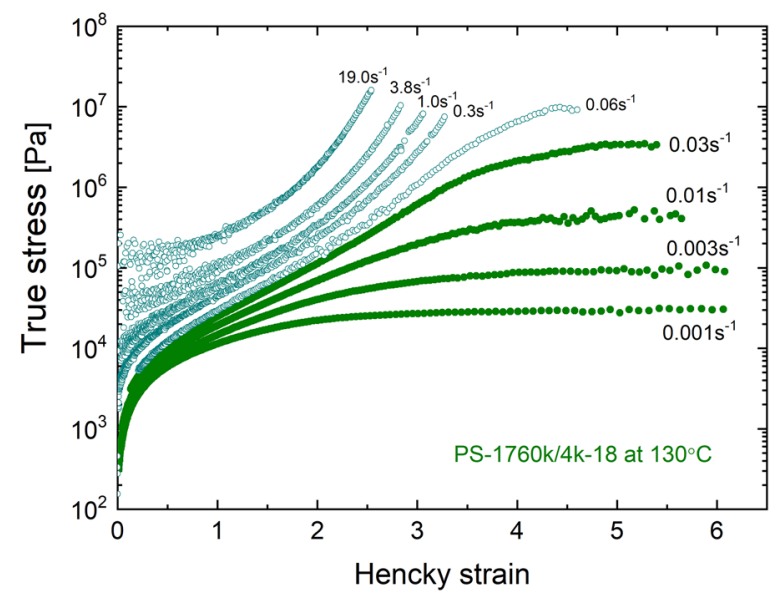

FIG. 3. The true stress as a function of Hencky strain for PS-1760k/4k-18 at different stretch rates at $130^{\circ} \mathrm{C}$. The plots for the stretch rates from $0.001 \mathrm{~s}^{-1}$ to $0.06 \mathrm{~s}^{-1}$ are taken from Ref. 27. 

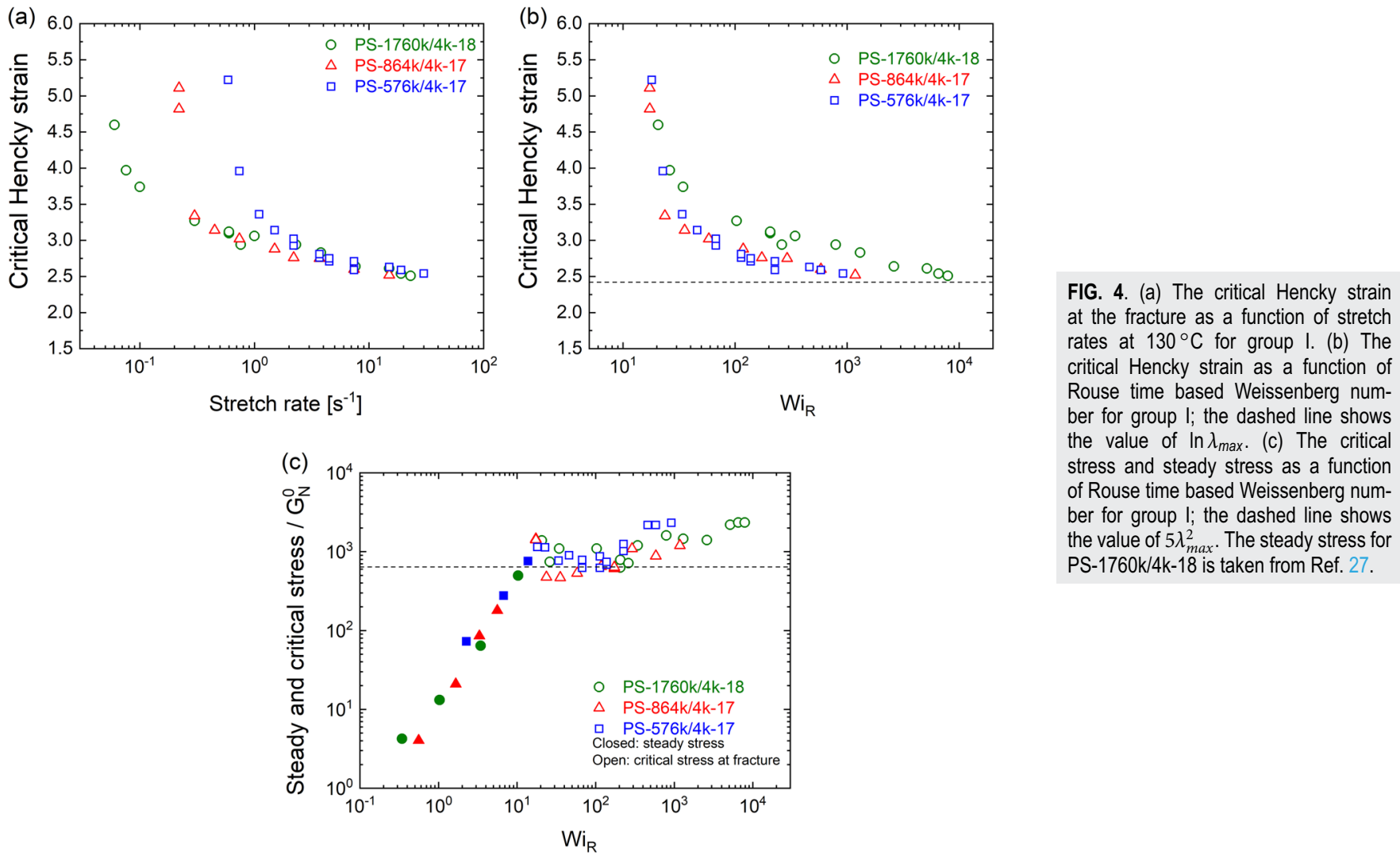
PS-1760k/4k-18 is taken from Ref. 27 .

independent of $Z$. We further plot the critical stress at the fracture as a function of the Weissenberg numbers for the three samples in Fig. 4(c) (shown as the open symbols). The steady stresses at lower $W i_{R}$ are also plotted in the figure (shown as the closed symbols). Here, all the stresses are normalized by the plateau modulus $G_{N}^{0}$; keep in mind, however, that the three samples have similar $G_{N}^{0}$. The values of $Z$ range from 6.7 to 22.8 for the samples. If the fracture occurs due to disentanglement, we would expect the sample with the lowest $Z$ is the easiest to disentangle, which leads to the lowest critical stress at the fracture. However, it is clear in Fig. 4(c) that the critical stress is independent of $Z$. Figures 4(b) and 4(c) suggest that the fracture happens at a length scale shorter than the entanglement strand, indicating that it originates from chain scission rather than rapid entanglement slipping. It should be noted that $Z$ is an average value and in the sample there are chains that have fewer entanglements than $Z$. The initiation of fracture could be triggered by such "weakest links" leading to disentanglement. But the major mechanism leading to fracture is probably not disentanglement since the critical strain and stress are not influenced by $Z$ as mentioned above.

In Fig. 4(c), the level of the critical stress is over 500 times higher than the plateau modulus, meaning that the polymer chains are highly stretched. In the elastic limit, the critical true stress at the fracture can be calculated as $\sigma_{c}=5 G_{N}^{0} \lambda_{c}^{2}$, where $\lambda_{c}$ is the critical stretch ratio of the polymer chains at the fracture. In Fig. 4(c), the dashed line shows the level of $5 \lambda_{\max }^{2}$, where $\lambda_{\max }=11.3$ for the samples in group I (see Table I). It can be seen that all the steady stresses are below the dashed line, while the critical stresses are of the same level, or slightly higher. This observation suggests that at the fracture the critical stretch ratio of the polymer chains, $\lambda_{c}$, approaches the theoretical maximum stretch ratio, $\lambda_{\max }$. The slightly higher stresses above the dashed line are probably due to glassy contribution; keep in mind that, here, we completely omit the contribution from the solvent OS-4k. The dashed line in Fig. 4(b) is calculated from $\epsilon_{c}$ $=\ln \lambda_{\max }$. It shows the limit of affine deformation when $\lambda_{\max }$ is approached.

\section{Critical strain and stress for group II (similar $Z$ and different $\boldsymbol{N}_{e}$ )}

Figure 5 shows the same plots as Figs. 4(b) and 4(c) but for the three samples in group II. For the melt PS-285k, at Weissenberg numbers of about $60-300$, steady stress seemed to be approached for about one Hencky strain unit, but then the samples ruptured. This might be due to the fact that the stretch rate is in the transition from necking to fracture, and necking may happen too fast to be controlled by the online control loop. It is therefore not clear whether such a rupture is due to elastic fracture or a failure of the online control scheme ${ }^{19}$ leading to fast necking. To clarify this point would require high-speed imaging to check the rupture process, which is not performed in this work. The strain and stress at such a rupture are plotted with the gray star symbols in Figs. 5(a) and 5(b). In Fig. 5(a), PS-864k/4k-33 and PS-285k seem to have the same critical 

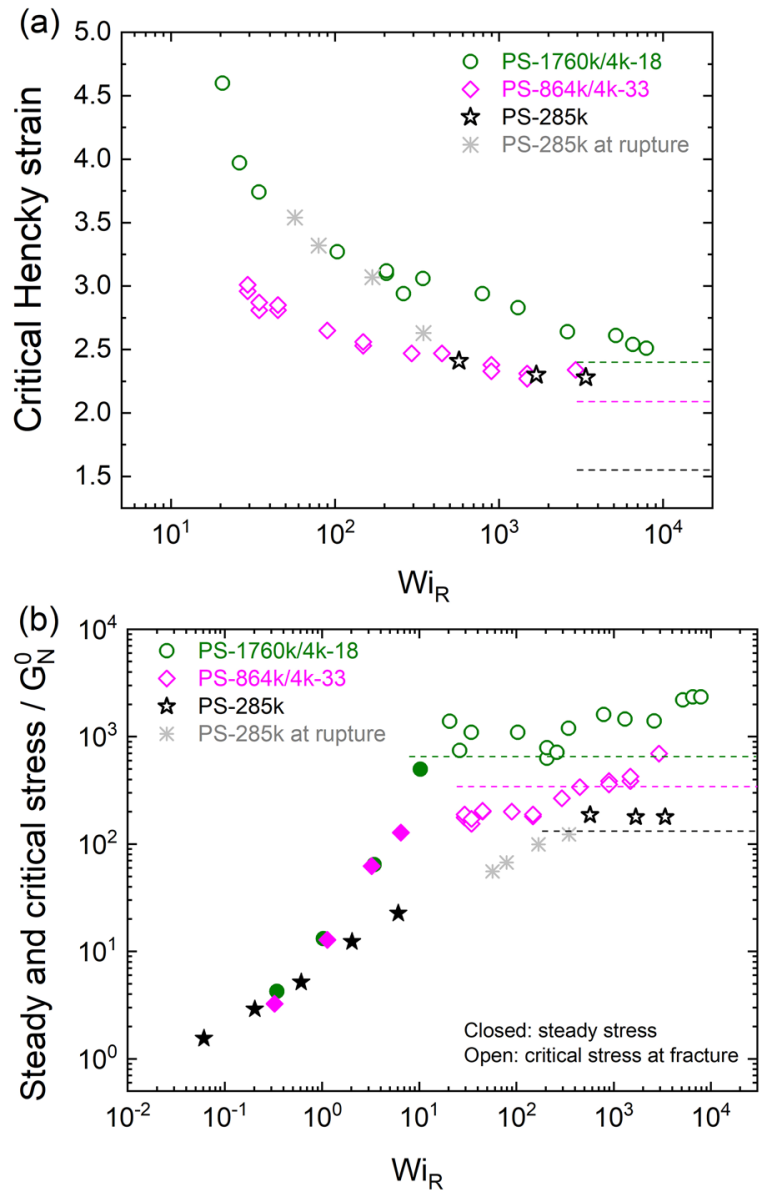

FIG. 5. (a) The critical Hencky strain as a function of Rouse time based Weissenberg numbers for group II; the dashed lines show the values of $\ln \lambda_{\max }$ for each sample. (b) The critical stress and steady stress as a function of Rouse time based Weissenberg numbers for group II; the dashed lines show the values of $5 \lambda_{\max }^{2}$ for each sample. The steady stress of PS-1760k/4k-18 and PS-285k is taken from Refs. 27 and 25, respectively; the steady stress of PS-900k/4k-33 in Ref. 27 is used here to represent the steady stress of PS-864k/4k-33. For PS-285k at Wi $\mathrm{i}_{\mathrm{R}}$ of $60-300$, it is not clear whether the sample at the rupture is due to elastic fracture or failure of the online control scheme leading to fast necking. Such points are presented in gray stars in both panels.

Hencky strain, while PS-1760k/4k-18 has a slightly higher critical Hencky strain. Therefore, it is not very clear whether the critical strain is $N_{e}$-dependent. By contrast, the critical stress in Fig. 5(b) shows a clear difference compared to the case in Fig. 4(c), meaning that it is indeed influenced by $N_{e}$. The dashed lines in Fig. 5(b) show the level of $5 \lambda_{\max }^{2}$ for each sample, which match the level of the critical stresses. This again suggests that the polymer chains approach their theoretical maximum stretch ratio $\lambda_{\max }$ at the fracture. The dashed lines in Fig. 5(a) show the limit of affine deformation for each sample when $\lambda_{\max }$ is reached. At high Weissenberg numbers, the two polystyrene solutions seem to approach the affine deformation, while the melt is still far away from the limit of affine deformation. This is probably due to the effect of more friction reduction for the melt compared to the solutions, ${ }^{32-34}$ which leads to faster relaxation for the polymer chains in the melt.

\section{CONCLUSIONS}

We have investigated the critical conditions at the fracture for two groups of nearly monodisperse linear polystyrene liquids in an extensional flow. We found that the dependence of critical strain on $N_{e}$ is not very clear, probably due to the complicated relationship between the macroscopic strain and the microscopic chain stretch. By contrast, the critical stress at the fracture is clearly independent of the number of entanglements per chain, but influenced by the number of Kuhn segments per entanglement strand. The observation indicates that the fracture in entangled polystyrene liquids originates more likely from chain scission, rather than rapid entanglement slipping.

However, the stress-strain measurements provide indirect information only; to confirm chain scission, other methods, e.g., with fluorescence, ${ }^{35}$ are required. The stress level at the fracture suggests that the polymer chains are highly stretched and approach the theoretical maximum stretch ratio. However, neutron scattering is needed to provide more direct information on how much the chain is stretched when fracture happens.

\section{ACKNOWLEDGMENTS}

Financial support from the Aage og Johanne Louis-Hansen Foundation is gratefully acknowledged. The author would like to thank Professor Ole Hassager from DTU Chemical Engineering for the useful discussions about interpretation of the experimental data.

\section{REFERENCES}

${ }^{1}$ A. A. Griffith, "The phenomena of rupture and flow in solids," Philos. Trans. R. Soc., A 221, 163-198 (1921).

${ }^{2}$ G. D. Zartman, S. Cheng, X. Li, F. Lin, M. L. Becker, and S.-Q. Wang, "How melt-stretching affects mechanical behavior of polymer glasses," Macromolecules 45, 6719-6732 (2012).

${ }^{3}$ Q. Huang, J. Madsen, L. Yu, A. Borger, S. R. Johannsen, K. Mortensen, and O. Hassager, "Highly anisotropic glassy polystyrenes are flexible," ACS Macro Lett. 7, 1126-1130 (2018).

${ }^{4}$ H. Tabuteau, S. Mora, G. Porte, M. Abkarian, and C. Ligoure, "Microscopic mechanisms of the brittleness of viscoelastic fluids," Phys. Rev. Lett. 102, 155501 (2009).

${ }^{5}$ X. Zhu and S.-Q. Wang, "Mechanisms for different failure modes in startup uniaxial extension: Tensile (rupture-like) failure and necking," J. Rheol. 57, 223-248 (2013).

${ }^{6}$ Q. Huang, N. J. Alvarez, A. Shabbir, and O. Hassager, "Multiple cracks propagate simultaneously in polymer liquids in tension," Phys. Rev. Lett. 117, 087801 (2016).

${ }^{7}$ J. H. Southern and D. R. Paul, "Elastic fracture of polystyrene solutions," Polym. Eng. Sci. 14, 560-566 (1974).

${ }^{8}$ J. H. Southern, R. L. Ballman, J. A. Burroughs, and D. R. Paul, "The polymer chain entanglement criterion for elastic fracture,” J. Polym. Sci., Polym. Lett. Ed. 16, 157-160 (1978).

${ }^{9}$ G. V. Vinogradov, "Viscoelasticity and fracture phenomenon in uniaxial extension of high-molecular linear polymers," Rheol. Acta 14, 942-954 (1975).

${ }^{10}$ A. Y. Malkin and G. V. Vinogradov, "Fracture of polymers in the visco-fluid state on stretching," Polym. Sci. U.S.S.R. 27, 245-257 (1985).

${ }^{11}$ J. Eggers and E. Villermaux, "Physics of liquid jets," Rep. Prog. Phys. 71, 036601 (2008).

${ }^{12}$ S. M. Fielding, "Criterion for extensional necking instability in polymeric fluids,” Phys. Rev. Lett. 107, 258301 (2011). 
${ }^{13}$ E. J. Hemingway, H. Kusumaatmaja, and S. M. Fielding, "Edge fracture in complex fluids," Phys. Rev. Lett. 119, 028006 (2017).

${ }^{14}$ A. Y. Malkin, A. Arinstein, and V. G. Kulichikhin, "Polymer extension flows and instabilities," Prog. Polym. Sci. 39, 959-978 (2014).

${ }^{15}$ A. Y. Malkin and C. J. S. Petrie, "Some conditions for rupture of polymer liquids in extension," J. Rheol. 41, 1-25 (1997).

${ }^{16}$ Y. Pomeau, "Brisure spontanée de cristaux bidimensionnels courbés," C. R. Acad. Sci. Paris 314 II, 553-556 (1992).

${ }^{17}$ D. Bonn, H. Kellay, and M. Prochnow, "Delayed fracture of an inhomogeneous soft solid," Science 280, 265-267 (1998).

${ }^{18}$ P. G. de Gennes, “Soft adhesives," Langmuir 12, 4497-4500 (1996).

${ }^{19}$ J. M. Román Marín, J. K. Huusom, N. J. Alvarez, Q. Huang, H. K. Rasmussen, A. Bach, A. L. Skov, and O. Hassager, "A control scheme for filament stretching rheometers with application to polymer melts," J. Non-Newtonian Fluid Mech. 194, 14-22 (2013).

${ }^{20} \mathrm{Y}$. Wang and S.-Q. Wang, "Salient features in uniaxial extension of polymer melts and solutions: Progressive loss of entanglements, yielding, non-Gaussian stretching, and rupture," Macromolecules 44, 5427-5435 (2011).

${ }^{21}$ M. H. Wagner, E. Narimissa, and Q. Huang, "On the origin of brittle fracture of entangled polymer solutions and melts," J. Rheol. 62, 221-233 (2018).

${ }^{22}$ S.-Q. Wang, "Letter to the Editor: Melt rupture unleashed by few chain scission events in fully stretched strands," J. Rheol. 63, 105-107 (2019).

${ }^{23}$ P. G. de Gennes, Scaling Concepts in Polymer Physics (Cornel University Press, Ithaca, New York, 1986).

${ }^{24} \mathrm{M}$. Doi and S. F. Edwards, The Theory of Polymer Dynamics (Clarendon Press, Oxford, 1986)

${ }^{25}$ Q. Huang, O. Mednova, H. K. Rasmussen, N. J. Alvarez, A. L. Skov, K. Almdal, and O. Hassager, "Concentrated polymer solutions are different from melts: Role of entanglement molecular weight," Macromolecules 46, 5026-5035 (2013).
${ }^{26}$ M. Baumgaertel, A. Schausberger, and H. H. Winter, "The relaxation of polymers with linear flexible chains of uniform length," Rheol. Acta 29, 400-408 (1990).

${ }^{27}$ Q. Huang, L. Hengeller, N. J. Alvarez, and O. Hassager, "Bridging the gap between polymer melts and solutions in extensional rheology," Macromolecules 48, 4158-4163 (2015).

${ }^{28} \mathrm{Q}$. Huang and O. Hassager, "Polymer liquids fracture like solids," Soft Matter 13, 3470-3473 (2017).

${ }^{29} \mathrm{M}$. van Gurp and J. Palmen, "Time-temperature superposition for polymeric blends," Rheol. Bull. 67, 5-8 (1998).

${ }^{30} \mathrm{Z}$. Qian and G. B. McKenna, "Expanding the application of the van GurpPalmen plot: New insights into polymer melt rheology," Polymer 155, 208-217 (2018).

${ }^{31}$ M. Rubinstein and R. H. Colby, Polymer Physics (Oxford University Press, Oxford, 2003).

${ }^{32}$ T. Yaoita, T. Isaki, Y. Masubuchi, H. Watanabe, G. Ianniruberto, and G. Marrucci, "Primitive chain network simulation of elongational flows of entangled linear chains: Stretch/orientation-induced reduction of monomeric friction," Macromolecules 45, 2773-2782 (2012).

${ }^{33}$ G. Ianniruberto, A. Brasiello, and G. Marrucci, "Simulations of fast shear flows of PS oligomers confirm monomeric friction reduction in fast elongational flows of monodisperse PS melts as indicated by rheooptical data," Macromolecules 45, 8058-8066 (2012).

${ }^{34}$ Y. Matsumiya, H. Watanabe, Y. Masubuchi, Q. Huang, and O. Hassager, "Nonlinear elongational rheology of unentangled polystyrene and poly(p-tertbutylstyrene) melts," Macromolecules 51, 9710-9729 (2018).

${ }^{35}$ E. Ducrot, Y. Chen, M. Bulters, R. P. Sijbesma, and C. Creton, "Toughening elastomers with sacrificial bonds and watching them break," Science 344, 186-189 (2014). 